\title{
MedienPädagogik
}

Zeitschrift für Theorie und Praxis der Medienbildung www.medienpaed.com

ISSN 1424-3636

Themenheft Nr. 40: CoViD-19 und die digitale Hochschulbildung. Irritationen, Einsichten und Programmatiken

Herausgegeben von Markus Deimann, Marios Karapanos und Klaus Rummler

\section{Hochschullehre im Pandemiebetrieb}

Wie Studierende in Sachsen das digitale Sommersemester erlebten

Marios Karapanos, Robert Pelz, Patrick Hawlitschek und Heinz-Werner Wollersheim

Zusammenfassung

Im Sommersemester 2020 beteiligten sich 3469 Studierende sächsischer Hochschulen an einer Onlinebefragung zum Studium während der Coronapandemie. Trotz Campusschliessungen konnten die Befragten ihr Studium weitgehend wie geplant fortführen. Während die technische Ausstattung der Studierenden eine Teilnahme an digitalen Lehrveranstaltungen zuliess, zeigten sich Defizite bei der Qualität der Internetverbindung. Im Vergleich zum Vorsemester wurden die Studienorganisation als moderat $(d=-0.48,95 \% \mathrm{Cl}[-0.52$, -0.45]), Lernaktivitäten als deutlich ( $d=-0.86,95 \% \mathrm{Cl}[-0.90,-0.82])$ schwerer empfunden. Auch die Konzentration auf das Studium fiel den Befragten schwerer als im Semester da$\operatorname{vor}(d=-0.69,95 \% \mathrm{Cl}[-0.72,-0.65])$. Mit den angebotenen Lernmedien zeigten sich die Befragten tendenziell zufrieden. Vor allem Learning-Management-Systeme, digitale Texte und Präsentationen dominierten das Lerngeschehen an den sächsischen Hochschulen. Fehlende soziale Interaktionen mit Peers und fehlende lernbezogene Interaktionen mit Dozentinnen und Dozenten wurden als besonders nachteilig erlebt. Zeit- und Kostenersparnisse sowie mehr Autonomie beim Lernen sind die grössten Vorteile des Studierens von zu Hause. Es zeigen sich keine Hinweise, dass leistungsschwache Studierende von den veränderten Studienbedingungen stärker nachteilig betroffen waren.

Higher education in pandemic mode. How students in Saxony experienced the digital summer semester

\begin{abstract}
In the Summer Semester of 2020, 3469 students enrolled in Saxon universities participated in an online survey concerning the study situation during the corona pandemic. The results show that, despite campus closures, the respondents were, on the whole, able to continue their studies as planned. While their technical equipment largely allowed participation in digital courses, not all students had a sufficiently stable internet connection. Compared to the previous semester, the respondents found it more difficult to organize their studies $(d=-0.48,95 \% \mathrm{Cl}[-0.52,-0.45])$ and to manage their learning activities $(d=0.86,95 \% \mathrm{Cl}$ [-0.90, -0.82]). It was also more difficult to concentrate on studying in general $(d=-0.69$, $95 \% \mathrm{Cl}[-0.72,-0.65])$. The respondents were mostly satisfied with the learning media offered. For the most part learning management systems, digital texts, and presentations
\end{abstract}


dominated the learning process at the universities in Saxony. The lack of social interactions with peers and learning-related interactions with lecturers were felt to be particularly disadvantageous. Savings in time and money, as well as more autonomy in learning, were considered the biggest advantages of studying from home. There is no indication that low-performing students were more affected negatively by the changed study conditions.

\section{Einführung}

Die Bedrohung durch SARS-CoV-2 stellt Bildungseinrichtungen weltweit auf eine enorme Belastungsprobe. Die deutschen Hochschulen reagierten im Sommersemester 2020 - wie Hochschulen in vielen Ländern - mit Campusschliessungen und einer nahezu vollständigen Verlagerung aller Lehraktivitäten in den digitalen Raum (Crawford u. a. 2020). Brachten bisherige Förderanstrengungen zum digitalen Lehren und Lernen in der Vergangenheit nur begrenzte Veränderungen im Hochschulalltag mit sich (Bond u. a. 2018; Persike und Friedrich 2016), erfassten die aus der Not beschlossenen Massnahmen nun nahezu sämtliche Aspekte von Studium und Lehre. Hochschullehrende und Studierende waren gleichermassen aufgefordert, neue technikvermittelte Formen des Lehrens, Lernens und Zusammenarbeitens zu finden und innerhalb kurzer Zeit einzusetzen. Damit bot sich mit dieser Ausnahmesituation gleichzeitig die Chance, das in einer solchen Breite noch nie praktizierte digitale Lehren und Lernen forschend zu begleiten, um einerseits auftretende Probleme zu identifizieren und praxisnahe Lösungen erarbeiten zu können, andererseits aber auch, um realistische Potenziale aufzuzeigen und zukünftige Forschungs- und Entwicklungsvorhaben daran auszurichten zu können.

An einer Vielzahl deutscher Hochschulen wurden im Sommersemester OnlineBefragungen unter Studierenden durchgeführt mit dem Ziel, sich ein genaueres Bild über deren allgemeine und studiumsbezogene Situation zu verschaffen. Vorab in der Presse veröffentlichte Ergebnisse deuten auf nicht zu vernachlässigende Unsicherheiten unter ihnen hin. Richter und Dunkhase (2020) berichten aus einer Befragung von 10000 Studierenden, dass etwa jedem Zehnten die Umstellung auf Online-Vorlesungen Sorgen bereitete. Nur etwa die Hälfte der Befragten wünschte sich eine Fortsetzung des Studiums einzig mit Online-Vorlesungen. Jeder Fünfte hätte lieber ein Semester pausiert. Gemäss einer Befragung an der Universität Würzburg mit über 3900 Teilnehmenden erwarteten $43 \%$ der Befragten negative Auswirkungen auf ihr Studium. Obwohl nur $26 \%$ angaben, Vorbehalte gegenüber dem Lernen mit digitalen Medien zu haben, befürchteten $76 \%$, dass das Studium dadurch unpersönlicher werde (Pauli, Neuderth, und Schuppert, 2020). Psychologisch orientierte Untersuchungen an Allgemeinbevölkerungsstichproben zeigten zudem eine Abnahme der allgemeinen Lebenszufriedenheit (Zacher und Rudolph 2020) und eine Zunahme von 
unspezifischem psychischen Distress (Sibley u. a. 2020). Von einem «normalen Semester, in dem ohne Hürden von zuhause studiert wird, war von Beginn an nicht auszugehen.

Vor dem Hintergrund variierender Rahmenbedingungen lassen sich die Ergebnisse der angeführten Befragungsstudien nicht ohne Weiteres auf den sächsischen Hochschulraum übertragen. So wurden in der Vergangenheit oft Initiativen zur Digitalisierung von Studium und Lehre, wie bspw. das Bildungsportal Sachsen oder der Virtuelle Campus Rheinland-Pfalz, hochschulübergreifend auf Ebene der Bundesländer organisiert.

Eine Standortbestimmung der neuen, digitalen Studienbedingungen scheint angesichts dessen ratsam. Neben praktisch orientierten Fragen etwa zum Fortgang, zu Voraussetzungen und zur Zufriedenheit beim technikvermittelten Studium sollen dabei auch forschungsorientierte Fragen zu interindividuellen Unterschieden adressiert werden.

Die reine Verfügbarkeit digitaler Lernangebote gewährleistet jedoch noch kein zufriedenstellendes und erfolgreiches Studieren. Entscheidend ist darüber hinaus, inwieweit Studierende auch über die notwendigen individuellen Voraussetzungen verfügen, um diese auch wahrnehmen zu können. Technikbereitschaft wird als generalisierte Disposition zum erfolgreichen Umgang mit Technik verstanden und setzt sich aus den Facetten Technikakzeptanz, -kompetenz und -kontrollüberzeugungen zusammen (Neyer, Felber, und Gebhardt 2012). Technikbereitschaft gilt als valider Prädiktor für Techniknutzung und könnte demnach Unterschiede in der Zufriedenheit mit technikvermitteltem Lernen, Studienorganisation und allgemein der Bewältigung des Studiums im Sommersemester erklären, das, durch zeitweisen Lockdown und Kontaktbeschränkungen bedingt, eine umfassende Nutzung von Technik zur Lösung alltäglicher Probleme und Aufgaben erforderte.

Befragungen aus dem Schulkontext zeigen, dass der Wechsel zum selbstorganisierten Lernen nicht allen Schülerinnen und Schülern gleichermassen gut gelingt. Wie aus den Daten des Schulbarometers hervorgeht, verbrachte ein grosser Teil der Schülerinnen und Schüler deutlich weniger Zeit in der Woche mit Lernen als vor den durch Corona bedingten Schulschliessungen. So gaben $18 \%$ der Befragten an, nur 9 Stunden in der Woche oder weniger gelernt zu haben (Huber und Helm 2020). Der Befund korrespondiert mit den Ergebnissen einer Elternbefragung, die ebenfalls auf eine deutliche Reduktion der Lernzeit hinweist (Wössmann u. a. 2020). Befürchtet wird zudem ein 'Schereneffekt), also eine Aufweitung der Leistungsunterschiede zwischen leistungsschwachen und -starken Schülerinnen und Schülern, der soziale Ungleichheiten verstärken könnte. Dass dieser auch die Hochschulen erreicht, ist zumindest nicht auszuschliessen. Die digitalen Angebote, die Studierende erreichen, haben zum Ziel, das Studium wie geplant zu ermöglichen. Unter den neuen Bedingungen ist es erwartbar, dass sich Prozesse der Studien- und Lernorganisation verändern. In welchem Ausmass, ist jedoch gegenwärtig unbekannt. 
So wünschenswert eine umfassende Betrachtung von Studium und Lehre unter den Bedingungen der Coronapandemie ist, so sehr zwingt die Methode Befragung zu einer Fokussierung auf einige wenige drängende Aspekte insbesondere dann, wenn sie online durchgeführt wird. Die Arbeit beschränkt sich daher auf folgende Fragestellungen:

1. In welchem Umfang gelingt es Studierenden in Sachsen, ihr Studium wie geplant fortzuführen?

2. In welchem Umfang verfügen sie über die notwendigen technischen und individuellen Voraussetzungen für ein digitales Studium von zuhause?

3. Welche Medientypen bestimmen die Lernpraxis und wie zufrieden sind Studierende mit diesen und anderen studienrelevanten Ressourcen ihrer Hochschule?

4. Wie gut schaffen es Studierende, unter den gegebenen Umständen Anforderungen ihres Studiums zu bewältigen?

5. Wie vorteilhaft oder nachteilig werden definierte Aspekte des digitalen Studiums wahrgenommen?

6. Welche Rolle spielen Technikbereitschaft und akademisches Leistungsniveau für einen erfolgreichen Wechsel zum digitalen Studieren von zuhause?

\section{Methode}

Die vorgestellten Befunde basieren auf Daten einer Onlinebefragung, die im Sommersemester 2020 durchgeführt wurde. Zur Rekrutierung von Teilnehmenden wurden die Studiendekaninnen und -dekane staatlicher Universitäten, Fach-, Kunst- und Musikhochschulen in Sachsen per E-Mail angeschrieben und darum gebeten, den Teilnahmeaufruf an die Studierenden der von ihnen verantworteten Studiengänge weiterzuleiten. Zusätzlich erfolgten Hinweise auf die Onlinebefragung in den SocialMedia-Kanälen einzelner Hochschulen. Nicht berücksichtigt wurden in der Befragung Studierende privater Hochschulen, von Stiftungshochschulen, von Hochschulen mit weniger als 200 Studierenden und von den Hochschulen der Sächsischen Polizei und Verwaltung.

Die Befunde basieren damit auf einer Selbstselektionsstichprobe. Die Adressatinnen und Adressaten entscheiden dabei selbst, ob sie dem öffentlichen Aufruf zur Teilnahme folgen oder nicht. Solche Stichproben kommen in der Umfrageforschung - gerade im Onlinebereich - häufig vor, sind aber ausdrücklich keine Zufallsauswahl, sondern bilden eine willkürliche Zusammenstellung. Selbstselektionsstichproben lassen deshalb nur - wenn überhaupt - sehr begrenzt Aussagen über die Grundgesamtheit zu. Solche Aussagen wären nur unter der durchaus problematischen Annahme zulässig, dass sich Teilnehmende und Nicht-Teilnehmende in ihrem Antwortverhalten statistisch nicht unterscheiden. Prinzipiell gilt aber, dass ein gewisses Mass an Selbstselektion bei nahezu allen empirischen Untersuchungen zu finden ist, da die 
Teilnahme in der Regel freiwillig erfolgt (Döring und Bortz 2016, 306). Das gilt also auch für Untersuchungen, bei denen die Stichprobe methodisch elaborierter bspw. durch das Ziehen aus Einschreibelisten gebildet wird. Aus den gegebenen Umständen während der Pandemie heraus war die Onlinebefragung mit öffentlichem Aufruf der einzig praktikable zeitnahe Zugang zum Feld. Die Befunde sind daher mit grosser Vorsicht zu interpretieren und mehr als begründete Vermutung denn als faktische Beschreibung zu lesen. Insbesondere Aussagen zur technischen Ausstattung, zur Zufriedenheit mit digitalen Lernmedien und anderen digitalen Hochschulressourcen sind behutsam zu behandeln, da der Aufruf zur Befragung über E-Mail und Social Media erfolgte und damit wahrscheinlich in grösserem Masse computer- und medienaffine Studierenden erreichte. Untersuchungen an populationsrepräsentativen Stichproben (Feierabend, Rathgeb, und Reuter 2019) und solche zur studienbezogenen Mediennutzung (Grosch und Gidion 2011; Karapanos und Fendler 2015) weisen jedoch auf eine insgesamt hohe Medienaffinität und nahezu flächendeckende Versorgung mit Computern und mobilen Endgeräten bei jungen Erwachsenen hin. Aus methodischer Sicht problematischer ist hingegen die Rekrutierung über die Studiendekaninnen und -dekane als Multiplikatoren, die wahrscheinlich zum Ausfall ganzer Studiengänge führte, wenn diese der Bitte um Weiterleitung des Teilnahmeaufrufs nicht nachkamen.

\subsection{Stichprobe}

Bis zum Stichtag am 16. Juli beteiligten sich 3469 Studierende an der Befragung. Bis auf 4 ordneten sich alle Befragten einer der in der Befragung aufgeführten Hochschulen zu. Die Ausschöpfungsquote, bezogen auf die Grundgesamtheit, lag bei etwa $3 \%$. Festzustellen ist eine stark erhöhte Beteiligung unter Studierenden der Universität Leipzig. Die Stichprobe weist also einen deutlichen Home Bias ${ }^{1}$ auf. Auch für die Studierenden der HTW Dresden und der Hochschule Mittweida lässt sich eine anteilig höhere Teilnahmebereitschaft feststellen. Hingegen sind die Technische Universitäten in Dresden und Chemnitz in der Befragung unterrepräsentiert (siehe Tabelle 1). Als mögliche Ursachen hierfür sind konkurrierende Befragungen und eine damit einhergehende «Befragungsmüdigkeit) unter den Studierenden in Betracht zu ziehen. $43 \%$ der Befragten studierten zum Befragungszeitpunkt in einem Studiengang, der mit dem Staatsexamen abschliesst. $37 \%$ der Befragten absolvierten einen Bachelorstudiengang. Master- und Diplomstudierende waren mit jeweils etwa $10 \%$ in der Stichprobe vertreten. Abbildung 1 zeigt die Verteilung über die verschiedenen Fächergruppen.

1 Home Bias ist eigentlich ein Begriff der Finanzwissenschaft und bezeichnet die Neigung von Anlegern, überproportional in Heimatmärkten zu investieren. Wir verwenden den Begriff hier für ein überproportionales Teilnahmeverhalten von Studierenden der eigenen Fakultät und Hochschule. 


\begin{tabular}{|l|c|c|c|c|}
\hline & \multicolumn{2}{|l|}{$\begin{array}{l}\text { Befragungsdaten Som- } \\
\text { mersemester 2020 }\end{array}$} & \multicolumn{2}{l|}{$\begin{array}{l}\text { Statistisches Landesamt } \\
\text { - Daten Wintersemester } \\
2018 / 19\end{array}$} \\
\hline Hochschule & Anzahl & in \% & Anzahl & in \% \\
\hline Hochschule für Bildende Künste Dresden & 18 & 0.5 & 505 & 0.5 \\
\hline $\begin{array}{l}\text { Hochschule für Grafik und Buchkunst } \\
\text { Leipzig }\end{array}$ & 16 & 0.5 & 558 & 0.6 \\
\hline Hochschule für Musik Dresden & 10 & 0.3 & 608 & 0.6 \\
\hline Hochschule für Musik und Theater Leipzig & 39 & 1.1 & 1129 & 1.1 \\
\hline $\begin{array}{l}\text { Hochschule für Technik und Wirtschaft } \\
\text { Dresden }\end{array}$ & 330 & 9.7 & 4.55 & 4.5 \\
\hline $\begin{array}{l}\text { Hochschule für Technik, Wirtschaft und } \\
\text { Kultur Leipzig }\end{array}$ & 152 & 4.5 & 6054 & 6 \\
\hline Hochschule Mittweida & 350 & 10.3 & 6900 & 6.8 \\
\hline Hochschule Zittau/Görlitz & 127 & 3.7 & 2911 & 2.9 \\
\hline $\begin{array}{l}\text { Technische Universität Bergakademie } \\
\text { Freiberg }\end{array}$ & 148 & 4.3 & 3924 & 3.9 \\
\hline Technische Universität Chemnitz & 138 & 4.0 & 10387 & 10.2 \\
\hline Technische Universität Dresden & 167 & 4.9 & 30829 & 30.4 \\
\hline Universität Leipzig & 1678 & 49.2 & 29061 & 28.7 \\
\hline Westsächsische Hochschule Zwickau & 236 & 6.9 & 3955 & 3.9 \\
\hline Gesamt & 3465 & 100 & 101371 & 100 \\
\hline
\end{tabular}

Tab. 1.: Stichprobe und Grundgesamtheit.

Da das statistische Landesamt Sachsens die Fächergruppen geringfügig anders erfasst, ist ein direkter Abgleich nicht ohne weiteres möglich. Auffällig ist der hohe Anteil von Lehramtsstudierenden, der auch den hohen Anteil an Teilnehmenden in Staatsexamensstudiengängen erklärt. Dies dürfte über die hohe Beteiligung der Universität Leipzig, an der die Mehrheit der sächsischen Lehramtsstudierenden einen Abschluss anstrebt, moderiert sein. Das Durchschnittsalter der Befragten lag bei 23.3 Jahren $(S D=5.1) .32 \%$ der Befragten waren männlich, $64 \%$ weiblich, $1 \%$ divers. Die übrigen $3 \%$ machten keine Angabe zum Geschlecht. Weitere studienstatistische Merkmale wie Studiengang, Fach- und Studiensemester oder soziodemographische Merkmale wie Familienstatus und Angaben zur Berufstätigkeit wurden nicht erhoben, um eine Deanonymisierbarkeit einzelner Fälle zu vermeiden. Um Aussagen über mögliche differentielle Effekte in Abhängigkeit des Leistungsniveaus der Befragten treffen zu können, wurden diese gebeten, sich selbst mittels 3-stufiger Rangskala im unteren, mittleren oder oberen Leistungsdrittel innerhalb ihres Studiengangs zu verorten. Selbstberichte zu Leistungsindikatoren, darunter auch der relative Rang, korrelieren hoch $\left(r_{\text {obs }}=.76,90 \% \mathrm{Cl}[.73, .79]\right)$ mit den Indikatoren selbst (Kuncel, Credé, und Thomas 2005). Die Analyse zeigt, dass leistungsschwache Studierende in der Stichprobe deutlich unterrepräsentiert sind. Sie machen nur $5 \%$ der Befragten 
aus. $31 \%$ ordneten sich dem oberen Leistungsdrittel zu, $55 \%$ dem mittleren. Neben dem Home Bias weist die Stichprobe damit also auch einen deutlichen Überhang an Teilnehmenden mit mittlerem Leistungsniveau auf.

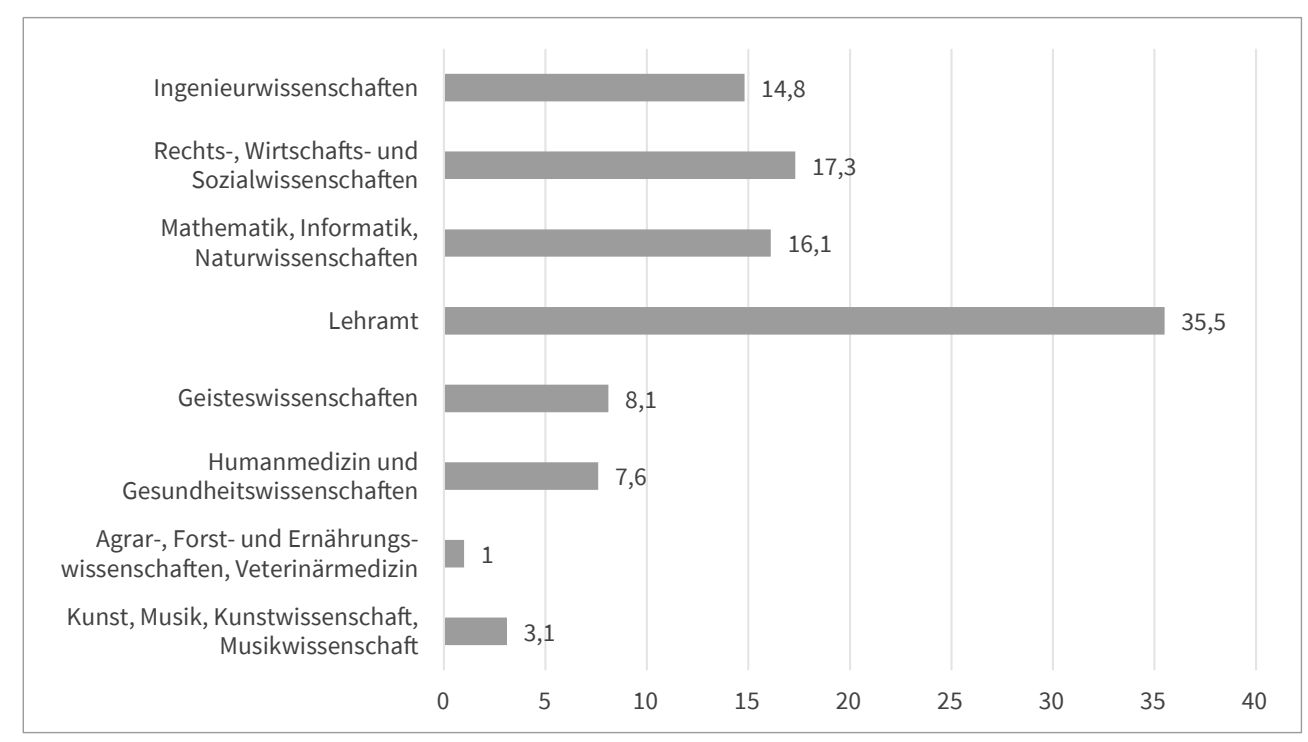

Abb. 1.: Stichprobenverteilung nach Fächergruppen (Alle Angaben in Prozent. Mehrfachnennungen waren möglich).

\subsection{Datenaufbereitung und Messmodell}

Angaben zu durchgeführten und ausgefallenen Lehrveranstaltungen wurden im Fragebogen numerisch erfasst. Werte grösser als 18 (99\%-Perzentil) wurden als Ausreisser behandelt und aus der Analyse ausgeschlossen. Alle übrigen Antwortskalen beinhalteten einen vordefinierten Wertebereich, sind also wenig anfällig gegenüber extremen Ausreissern. Für die meisten Fragen kamen 5-stufige uni- bzw. bipolare Ratingskalen zum Einsatz, die in der Regel von -2 bis +2 reichten. Werte nahe 2 bedeuten eine hohe Merkmalsausprägung (Bsp.: «stimme voll zu»), während Werte von -2 die Gegenkategorie (Bsp.: «stimme nicht zu») darstellen. Zur besseren Veranschaulichung werden im Ergebnisteil bei den Voraussetzungen für das digitale Studium die jeweils positiven (also 1 und 2) und die negativen (also -1 und -2) Antwortoptionen zu jeweils einer Kategorie zusammengefasst. Neben den deskriptiven Massen zu Studium und Mediennutzung wurden etablierte Instrumente zur Erfassung von Lernaktivitäten und Studienorganisation aus dem Messinstrument für die Wahrnehmung von Studienanforderungen (Jänsch und Bosse 2018), Technikakzeptanz, -kompetenz und -kontrolle (Kurzskala zur Erfassung von Technikbereitschaft; Neyer, Felber, und Gebhardt, 2012) und 4 Items der System Usability Scale (Brooke 1996) zur Erfassung der Gebrauchstauglichkeit des meistgenutzten Learning-Management-Systems in den Fragebogen aufgenommen. 


\begin{tabular}{|l|c|c|c|r|}
\hline & $M$ & $S D$ & Cronbachs $\alpha$ & $95 \% \mathrm{Cl}$ \\
\hline Technikkontrolle & 0.53 & 0.80 & .72 & $.70, .74$ \\
\hline Technikkompetenz ${ }^{+}$ & 0.87 & 1.01 & .87 & $.86, .88$ \\
\hline Technikakzeptanz & 0.09 & 1.04 & .85 & $.84, .86$ \\
\hline System Usability Scale & 69.2 & 24.4 & .85 & $.84, .86$ \\
\hline Studienorganisation & -0.38 & 0.79 & .76 & $.74, .77$ \\
\hline Lernaktivitäten & -0.80 & 0.93 & .83 & $.82, .84$ \\
\hline
\end{tabular}

Tab. 2.: Mittelwerte, Standardabweichungen und interne Konsistenz (Anmerkung. ${ }^{+}$invertiert).

Die faktoranalytische Überprüfung mittels konfirmatorischer Faktorenanalyse zeigt eine gute Passung $\left(\mathrm{X}^{2}=1393.3, d f=237, p<.001, \mathrm{CFI}=.965\right.$, RMSEA $=.038$, SRMR $=.036)$ zwischen Messmodell und empirischer Datenstruktur. Zur Bedeutung und Bewertung von Modellfit-Indizes sei auf Hu und Bentler (1999) verwiesen. Bis auf ein Item liegen alle standardisierten Faktorladungen bei .50 oder darüber. Abbildung 2 zeigt das Messmodell graphisch. Die Items zur Technikkompetenzskala sind negativ formuliert («Im Umgang mit moderner Technik habe ich oft Angst zu versagen»). Sie misst also vielmehr Technikinkompetenz. Zur einfacheren Interpretation wurde diese deshalb invertiert.

Die Reliabilität der sechs Skalen geschätzt mittels Cronbachs a rangiert auf akzeptablem bis sehr gutem Niveau zwischen .72 und .87. Scores für Studienanforderungen, Technikbereitschaft und System Usability werden nur dann berechnet, wenn von einer Skala mindestes drei Items beantwortet wurden. Das Scoring erfolgt durch Mittelwertbildung, was einer Imputation der fehlenden Werte durch den Mittelwert der vorhanden Werte entspricht (valid mean substitution). Der Wertebereich der Scores liegt zwischen -2 und +2 . Mit den 4 Items der System Usability Scale wird in ähnlicher Weise verfahren. Es wird lediglich eine zusätzliche Skalentransformation auf den Wertebereich 0 bis 100 vorgenommen, um eine Vergleichbarkeit mit Referenzwerten herstellen zu können. Alle Skalenparameter sind Tabelle 2 zu entnehmen. 


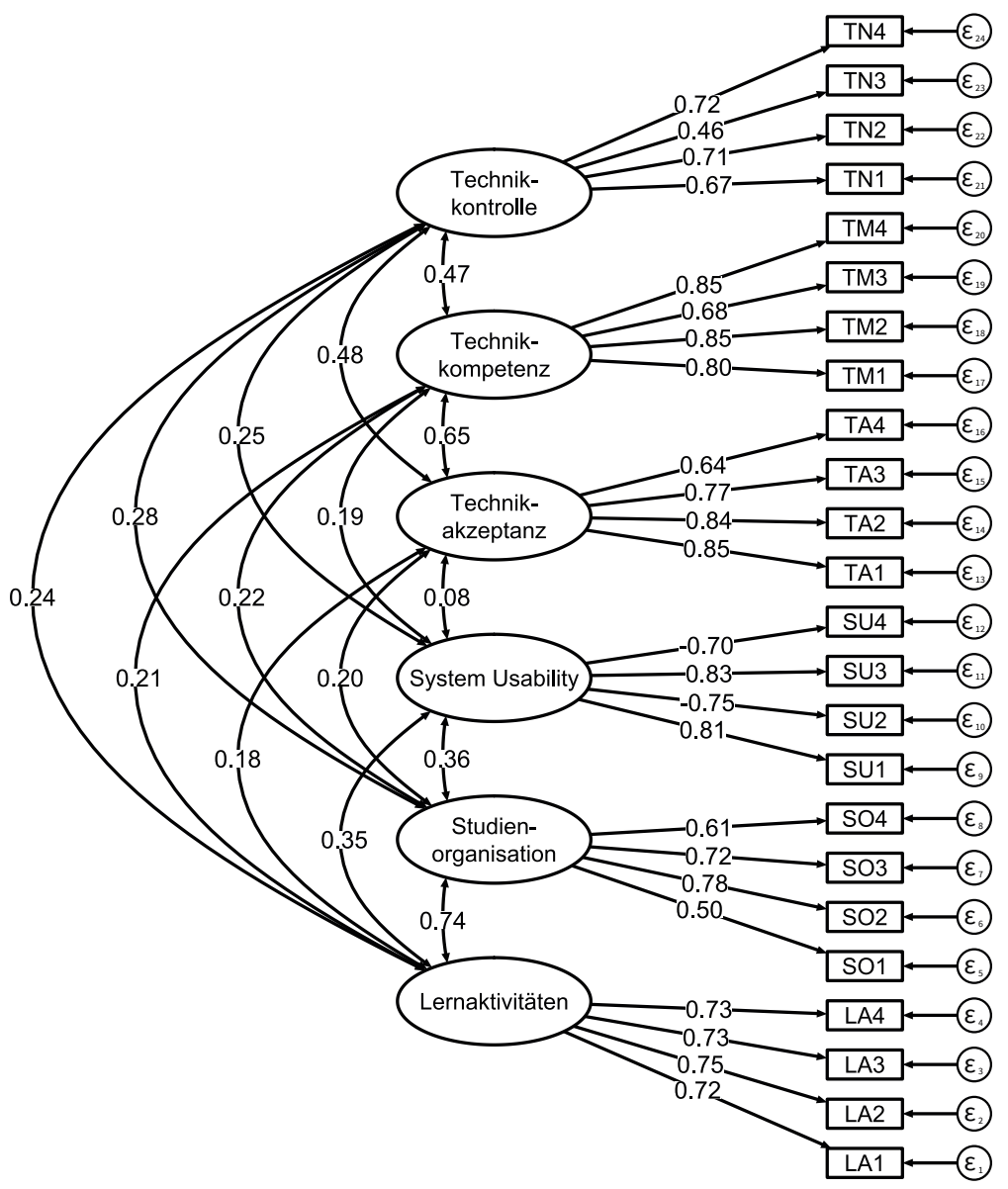

Abb. 2.: Messmodell mit standardisierten Faktorladungen und Faktorkorrelationen.

\section{Ergebnisse}

\subsection{Durchführung von Lehrveranstaltungen}

Trotz der Coronakrise war es den Studierenden im Wesentlichen möglich, ihr Studium fortzuführen. Im Mittel planten sie neun Veranstaltungen $(M=9.04, S D=4.18)$ im Sommersemester 2020 zu besuchen. An den meisten der von ihnen gewählten Veranstaltungen, also Vorlesungen, Seminaren, Laborpraktika usw., konnten sie wie geplant teilnehmen. Im Durchschnitt fanden sechs bis sieben Veranstaltungen planmässig statt $(M=6.34, S D=3.37)$, eine bis zwei starteten verspätet $(M=1.50$, $S D=2.34)$ und im Mittel fiel für jede(n) Befragte(n) nur etwa eine Veranstaltung ganz aus $(M=1.19, S D=1.67)$. Den sächsischen Hochschulen gelang es, die Lehre auch ohne Präsenzveranstaltungen weitgehend aufrechtzuerhalten. 


\subsection{Voraussetzungen für das digitale Studium}

Befragt nach den Voraussetzungen für das digitale Studium zeigt sich ein gemischtes Bild. Zwar haben nahezu alle Befragten Zugriff auf ein eigenes internetfähiges Endgerät (96\%). Aber nur drei von vier (76\%) gaben an, über eine stabile Internetverbindung zu verfügen. ${ }^{2}$ Ist eine solche vorhanden, ist sie in der Regel aber auch schnell genug für datenintensive Anwendungen wie Videotelefonie, Livestreaming oder den Download grosser Dateien (72 \%). Ein Mangel offenbart sich auch bei Peripheriegeräten wie Webcam, Mikrophon oder einem ausreichend grossen Bildschirm. Etwa jede( $r$ ) Siebente $(14 \%)$ meldete hier Defizite in der eigenen Ausstattung an. Bei der Software sieht es ähnlich aus. Auch hier verfügen etwa $13 \%$ der Befragten nicht über die notwendigen Programme, etwa zur Dokumentenbearbeitung oder der Erstellung von Präsentationen.

Dozentinnen und Dozenten scheinen allerdings überwiegend von einer ausreichenden Ausstattung unter Studierenden auszugehen. So gaben die Befragten an, dass im Mittel nur eine( $r)$ von sechs $(M=17 \%, S D=25 \%)$ ihrer Dozentinnen und Dozenten bei ihnen die Verfügbarkeit der notwendigen technischen Hilfsmittel nachgefragt habe. Bei etwa der Hälfte der Befragten ( $47 \%$ ) hatte sich keine(r) nach den vorhandenen technischen Voraussetzungen erkundigt.

Selbst Medien wie Screencasts, Podcasts oder Videoclips zu produzieren, traut sich mehr als die Hälfte der Befragten (54\%) nicht zu. Als Alternative zur Seminararbeit oder schriftlichen Prüfung kommen diese Formate zumindest in der Breite also nicht in Frage. Treten Probleme auf, so sehen sich viele der Befragten damit alleingelassen. So gab etwa jede(r) Dritte (35\%) an, nicht zu wissen, an wen er oder sie sich bei fachlichen, technischen oder organisatorischen Fragen wenden kann.

\subsection{Zufriedenheit mit studienrelevanten Hochschulressourcen}

Die Befragten zeigten sich überwiegend zufrieden mit studienrelevanten Ressourcen ihrer Hochschulen. Sieben von zehn Befragten (69\%) waren (sehr) zufrieden mit der Erreichbarkeit der Dozentinnen und Dozenten, (sehr) unzufrieden war hingegen nur etwa jede( $r$ ) Siebente $(15 \%)$. Am wenigsten zufrieden waren Studierende mit der Verfügbarkeit fachspezifischer Literatur. Hierin spiegelt sich offenbar der Umstand, dass Hochschulbibliotheken lange Zeit ganz geschlossen waren und erst später im Semester wieder öffneten. Die Häufigkeitsverteilungen zur Zufriedenheit sind Abbildung 3 zu entnehmen.

2 Die Items wurden im Fragenbogen mittels 5-stufiger unipolarer Ratingskalen von -2 (stimme nicht zu) bis +2 (stimme voll zu) beantwortet. Hier und im Folgenden werden jeweils die positiven Antwortkategorien als Zustimmung, die negativen Antwortkategorien als Ablehnung zusammengefasst (siehe Abschnitt 2.2). 


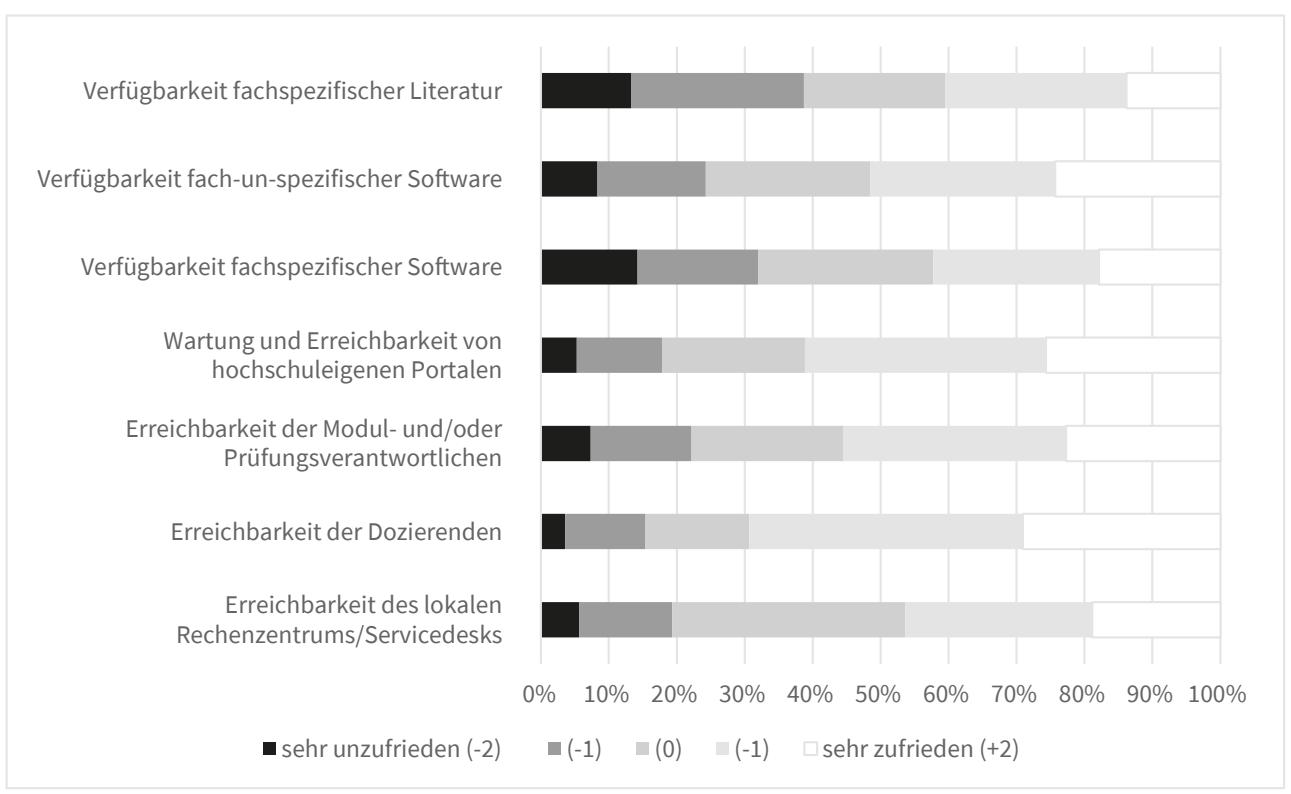

Abb. 3.: Zufriedenheit mit studienrelevanten Hochschulressourcen.

\subsection{Endgerätenutzung und technische Ausstattung}

Neun von zehn Studierenden (90\%) nutzten primär PC oder Laptop für das Studium zuhause. Tablets ( $8 \%$ ) und Smartphones ( $2 \%$ ) waren für eine Minderheit das am häufigsten genutzte Lernwerkzeug. Andere internetfähige Endgeräte (0.2 \%) spielten praktisch keine Rolle.

\subsection{Einsatz digitaler Medien in Lehrveranstaltungen}

Obwohl eine gewisse Variabilität bei den eingesetzten digitalen Lernmedien beobachtbar ist, dominierten einige wenige Medientypen das Lerngeschehen im abgelaufenen Sommersemester (siehe Abbildung 4). 


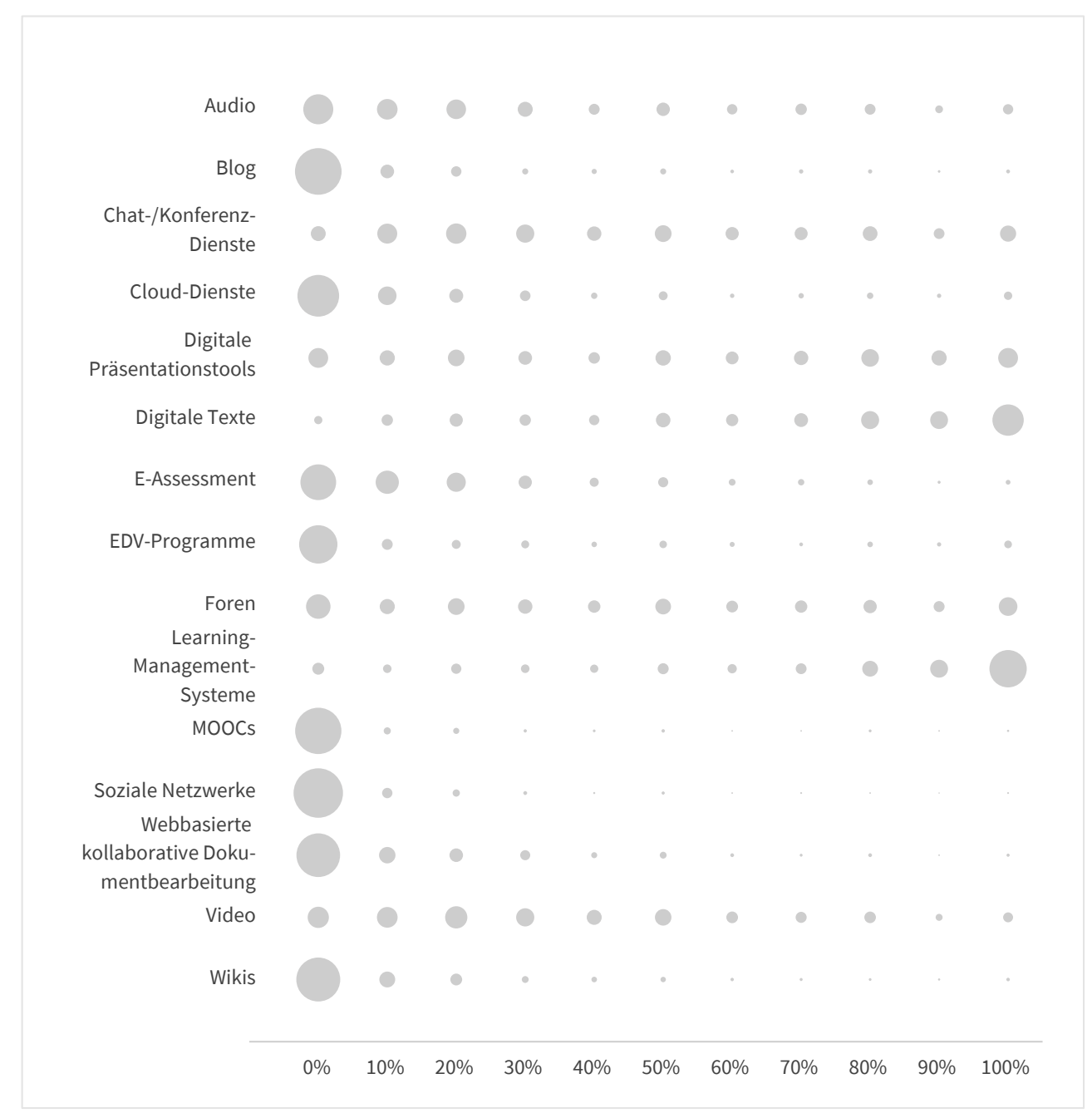

Abb. 4.: Einsatz digitaler Medien in Lehrveranstaltungen / Häufigkeitsverteilung der Antworten auf die Frage «Bitte geben Sie an, bei wie viel Prozent Ihrer Lehrveranstaltungen nachfolgend genannte digitale Medien und Anwendungen derzeit von Dozenten eingesetzt werden»).

Die Befragten gaben an, dass im Mittel in $71 \%(S D=31 \%)$ ihrer Lehrveranstaltungen digitale Texte, in $51 \%(S D=35 \%)$ digitale Präsentationen und in $43 \%(S D=31 \%)$ Chat- und Konferenzdienste eingesetzt wurden. Mit kollaborativen Werkzeugen wie webbasierter Dokumentenverarbeitung ( $M=7 \%, S D=14 \%$ ) oder Wikis ( $M=5 \%$, $S D=14 \%$ ) hatten die Studierenden deutlich seltener Kontakt. Die Auswahl der abgefragten Medientypen orientierte sich an Persike und Friedrich (2016). 


\subsection{Zufriedenheit mit digitalen Medien in Lehrveranstaltungen}

Neben der Einsatzhäufigkeit wurden die Studierenden auch nach der Zufriedenheit mit den digitalen Medien in ihrem Studium befragt (siehe Abbildung 5). Auf einer Skala von -2 (sehr unzufrieden) bis +2 (sehr zufrieden) erzielten die meisten der Angebote positive Mittelwerte. Mehrheitlich negativ wurden nur MOOCs $(M=-0.31$, $S D=1.02)$, Blogs $(M=-0.28, S D=1.04)$, Soziale Netzwerke $(M=-0.23, S D=1.16)$, Wikis $(M=-0.15, S D=1.09)$ und allgemein EDV-Software $(M=-0.02, S D=1.14)$ bewertet. Interessant an dieser Zusammenstellung ist die Tatsache, dass mit Blogs, Sozialen Netzwerken und Wikis gleich drei Medientypen vertreten sind, die eine produktive Eigenarbeit der Studierenden voraussetzen. Gleichsam populär und häufig im Einsatz sind Learning-Management-Systeme $(M=0.93, S D=1.03)$, digitale Präsentationen $(M=0.74, S D=1.07)$ sowie Chat- und Konferenzdienste $(M=0.56, S D=1.15)$. Ausbaupotential haben Audio $(M=0.72, S D=1.14)$ und Video $(M=0.88, S D=1.08)$. Beide erzielen hohe Zufriedenheitswerte, werden aber in der Lehre - vermutlich aufgrund hoher Produktionsaufwände und -hürden - noch vergleichsweise selten eingesetzt.

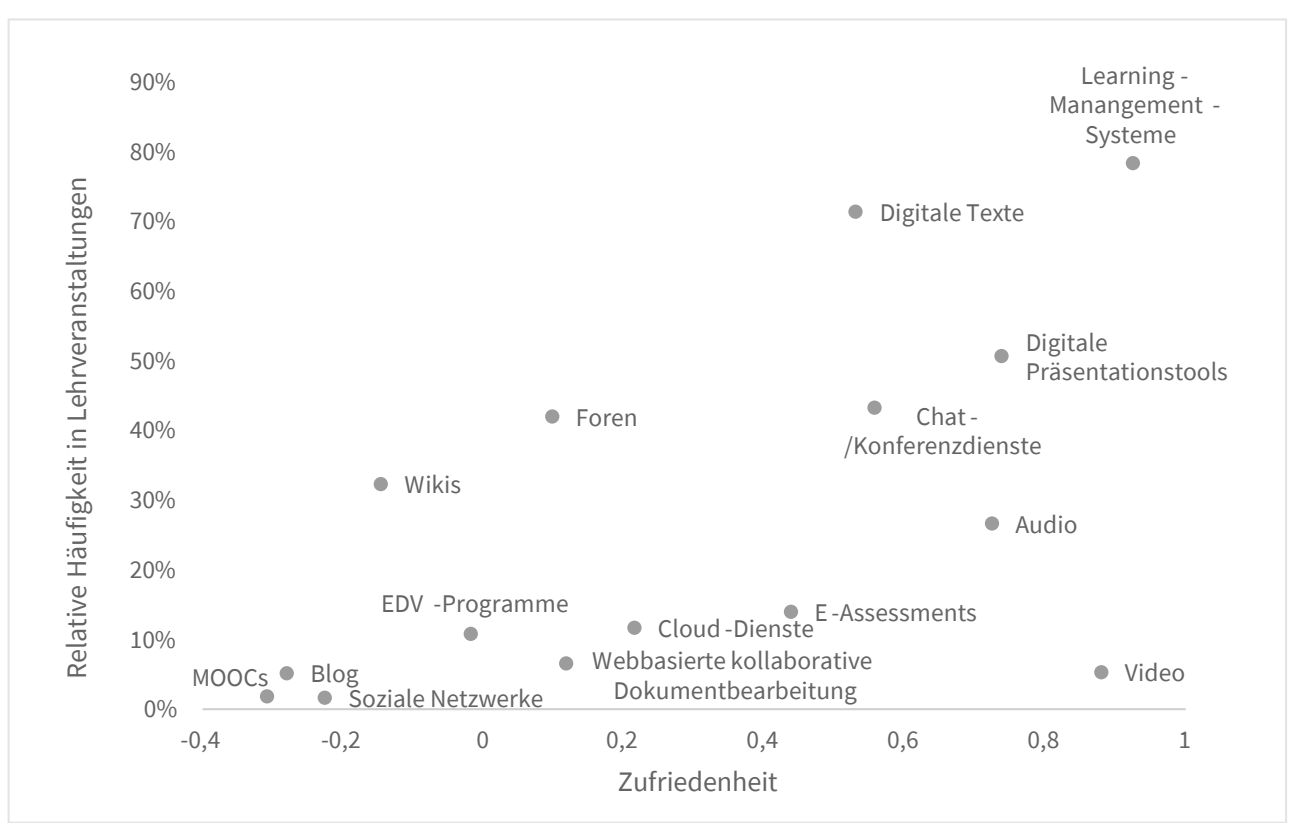

Abb. 5.: Einsatz von und Zufriedenheit mit digitalen Medien (Zufriedenheitsskala von -2 [sehr unzufrieden] bis +2 [sehr zufrieden]).

Die für jeden Medientyp angegebenen Zufriedenheitswerte lassen sich durch Mittelwertbildung zu einem Zufriedenheitsindex aggregieren $(M=0.56, S D=0.75)$. Dabei zeigen sich Unterschiede zwischen den Leistungsgruppen $(F(2,3095)=3.877$, $p=.021)$. Befragte des unteren Leistungsdrittels $(M=0.40, S D=0.90)$ waren mit den angebotenen Lernmedien etwas unzufriedener als jene des mittleren $(M=0.57$, 
$\left.S D=0.72, t(2040)=2.841, p_{\text {Holm }}=.015, d=0.23,95 \% \mathrm{Cl}[0.07,0.39]\right)$ und oberen Drittels $\left(M=0.56, S D=0.75, t(1215)=2.432, p_{\text {Holm }}=.030, d=0.23,95 \% \mathrm{Cl}[0.04,0.37]\right)$. Mittleres und oberes Terzil unterschieden sich nicht voneinander $(t(2935)=0.228$, $\left.p_{\text {Holm }}=.819, d=0.01,95 \% \mathrm{Cl}[-0.07,0.08]\right)$.

\subsection{Nutzung von Learning-Management-Systemen}

Learning-Management-Systeme (LMS) nutzten die Studierenden in etwa 4 von 5 Veranstaltungen ( $M=78 \%, S D=31 \%)$. Nur $5 \%$ der Befragten gaben an, gar kein LMS genutzt zu haben. Während $53 \%$ vorwiegend mit Moodle arbeiteten, nutzten $39 \%$ das an den meisten Hochschulen Sachsens etablierte OPAL. In diesen Zahlen spiegelt sich in hohem Mass der Überhang an Befragungsteilnehmenden der Universität Leipzig, an der in erster Linie auf eine eigene Moodle-Instanz gesetzt wird. Ausserhalb der Universität überwiegt jedoch der Anteil von OPAL mit $77 \%$ gegenüber dem von Moodle mit $13 \%$, wenn auch weniger deutlich als zu erwarten. Offenbar geben einige Fakultäten in Sachsen eigenen Moodle-Instanzen vor dem zentral durch das Bildungsportal Sachsen bereitgestellten OPAL den Vorzug. Die Gebrauchstauglichkeit (Usability) der Systeme wurde mittels 4 Items («Ich fühle mich bei der Benutzung des Learning-Management-Systems sehr sicher.») ${ }^{3}$ aus der System Usability Scale (SUS, Brooke, 1996; dt. Übersetzung siehe Rauer 2011) geschätzt. Mit SUS-Scores von 67.4 $(S D=24.8)$ für OPAL und $70.7(S D=23.5)$ ergibt sich zwar eine statistisch signifikante Diskrepanz $(t(2943)=3.579, p<.001, d=0.13,95 \% \mathrm{Cl}[0.06,0.21])$, die aber gemessen an der Effektstärke praktisch bedeutungslos ist. Beide Systeme werden als vergleichbar gebrauchstauglich eingeschätzt. Zwar gelten SUS-Scores dieser Grössenordnung als noch akzeptabel (Bangor, Kortum, und Miller 2009). Kommerzielle Webwerkzeuge wie der E-Mail-Dienst Gmail (83.5) oder die Website des Onlinehändlers Amazon (81.8) erzielen in Vergleichsstudien allerdings erheblich bessere Werte (Kortum und Bangor 2013). In diesem Punkt sind sowohl Moodle als auch OPAL also noch ausbaufähig.

\subsection{Anforderungen des Studiums}

Im Vergleich zum Vorsemester fiel es den befragten Studierenden deutlich schwerer, wesentlichen Anforderungen ihres Studiums gerecht zu werden. Erfasst wurden die Anforderungsbereiche Lernaktivitäten (4 Items, Beispiel: «... die Menge an Lernstoff zu bewältigen, z. B. semesterbegleitende Aufgaben oder Lektüren») und Studienorganisation (4 Items, Beispiel: «... passende Informations- und Beratungsangebote zu finden, z. B. Ansprechpersonen finden») mit den gleichnamigen Skalen des Messinstruments

3 Weitere Items: «Ich empfinde das Learning-Management-System als unnötig komplex.» «Ich kann mir vorstellen, dass die meisten Menschen schnell lernen, das Learning-Management-System zu beherrschen.», "Ich empfinde die Bedienung des Learning-Management-Systems als sehr umständlich.» 
für die Wahrnehmung von Studienanforderungen (Jänsch und Bosse 2018). Die Befragten wurden aufgefordert einzuschätzen, ob die ihnen vorgelegten Anforderungen für sie schwieriger oder leichter als im vorangegangenen Semester zu bewältigen waren. Einstichproben-t-tests weisen auf signifikante Unterschiede von der Skalenmitte $(M=0)$ hin. Sowohl die Lernaktivitäten $(M=0.78, S D=0.91, t(3398)=-49.922, p<.001$, $d=-0.86,95 \% \mathrm{Cl}[-0.90,-0.82])$ als auch die Studienorganisation $(M=-0.38, \mathrm{SD}=0.78$, $t(3151)=-27.091, p<.001, d=-0.48,95 \% \mathrm{Cl}[-0.52,-0.45])$ wurden als deutlich schwerer wahrgenommen. Der schwächere Effekt bei der Studioorganisation ist gut mit der Tatsache in Einklang zu bringen, dass zahlreiche Aspekte der Studienorganisation wie Kurswahl oder die Recherche von Informations- und Beratungsangeboten auch schon vorher weitgehend digital realisiert wurden. Beide Skalen korrelieren hoch $(r(3144)=.59,95 \% \mathrm{Cl}[.56, .61])$. Wer Schwierigkeiten mit der Studienorganisation hat, hat also typischerweise auch Schwierigkeiten mit dem Lernen und vice versa. Das Leistungsniveau scheint hingegen keine Rolle zu spielen. Weder bei den Lernaktivitäten noch bei der Studienorganisation zeigen sich Unterschiede zwischen Studierenden aus dem unteren, mittleren und oberen Leistungsdrittel (siehe Tabelle 3).

\begin{tabular}{|c|c|c|c|c|c|c|}
\hline & \multicolumn{3}{|c|}{ Leistungsdrittel } & \multicolumn{3}{|c|}{ ANOVA } \\
\hline & unteres & mittleres & oberes & $F$ & $d f$ & $p$ \\
\hline Lernaktivität & $\begin{array}{c}-0.81 \\
(1.04)\end{array}$ & $\begin{array}{l}-0.80 \\
(0.92)\end{array}$ & $\begin{array}{l}-0.76 \\
(0.89)\end{array}$ & 0.602 & 3142 & .548 \\
\hline Studienorganisation & $\begin{array}{l}-0.44 \\
(0.85)\end{array}$ & $\begin{array}{l}-0.37 \\
(0.78)\end{array}$ & $\begin{array}{l}-0.39 \\
(0.77)\end{array}$ & 0.588 & 2919 & .556 \\
\hline
\end{tabular}

Tab. 3.: Mittelwerte der Skalen Lernaktivität und Studienorganisation nach Leistungsdritteln (Standardabweichungen in Klammern notiert).

\subsection{Technikakzeptanz, -kompetenz und -kontrolle}

Technikakzeptanz, -kompetenz und -kontrolle korrelieren erwartungskonform auf moderatem bis hohem Niveau, zeigen aber nur schwache Zusammenhänge $(r<.19)$ mit der Bewältigung von Studienanforderungen oder der Zufriedenheit mit digitalen Medien im Studium (siehe Tabelle 4).

\begin{tabular}{|l|c|c|c|c|c|}
\hline & $1)$ & $2)$ & $3)$ & $4)$ & 5) \\
\hline 1) Technikakzeptanz & - & & & & \\
\hline 2) Technikkompetenz & $.52^{\star \star \star}$ & - & & & \\
\hline 3) Technikkontrolle & $.37^{\star \star \star}$ & $.35^{\star \star \star}$ & - & & \\
\hline 4) Lernaktivitäten & $.15^{\star \star \star}$ & $.17^{\star \star \star}$ & $.16^{\star \star \star}$ & - & \\
\hline 5) Studienorganisation & $.16^{\star \star \star}$ & $.17^{\star \star \star}$ & $.18^{\star \star \star}$ & $.59^{\star \star \star}$ & - \\
\hline 6) Zufriedenheitsindex & $.06^{\star \star \star}$ & $.11^{\star \star \star}$ & $.18^{\star \star \star}$ & $.40^{\star \star \star}$ & $.45^{\star \star \star}$ \\
\hline
\end{tabular}

Tab. 4.: Pearson-Korrelationen zwischen Technikbereitschaft, Studienanforderungen und Zufriedenheit mit digitalen Lernmedien $\left.{ }^{* * *} \mathrm{p}<.001\right)$. 
Die Technikkompetenzskala weist eine stark rechtssteile Verteilung mit gut erkennbarem Deckeneffekt auf $(M=0.90, S D=1.00$, Skewness $=0.81, S E=0.042)$. Die Befragten schreiben sich also im Mittel ein sehr hohes Mass an Technikkompetenz zu, die damit für das Studium offenbar keine limitierende Ressource bildet. Entsprechend unkritisch sind die Technikkompetenzunterschiede zwischen den Leistungsterzilen zu bewerten. Technikakzeptanz und -kontrollüberzeugungen sind hingegen vom akademischen Leistungsniveau unabhängig (siehe Tabelle 5).

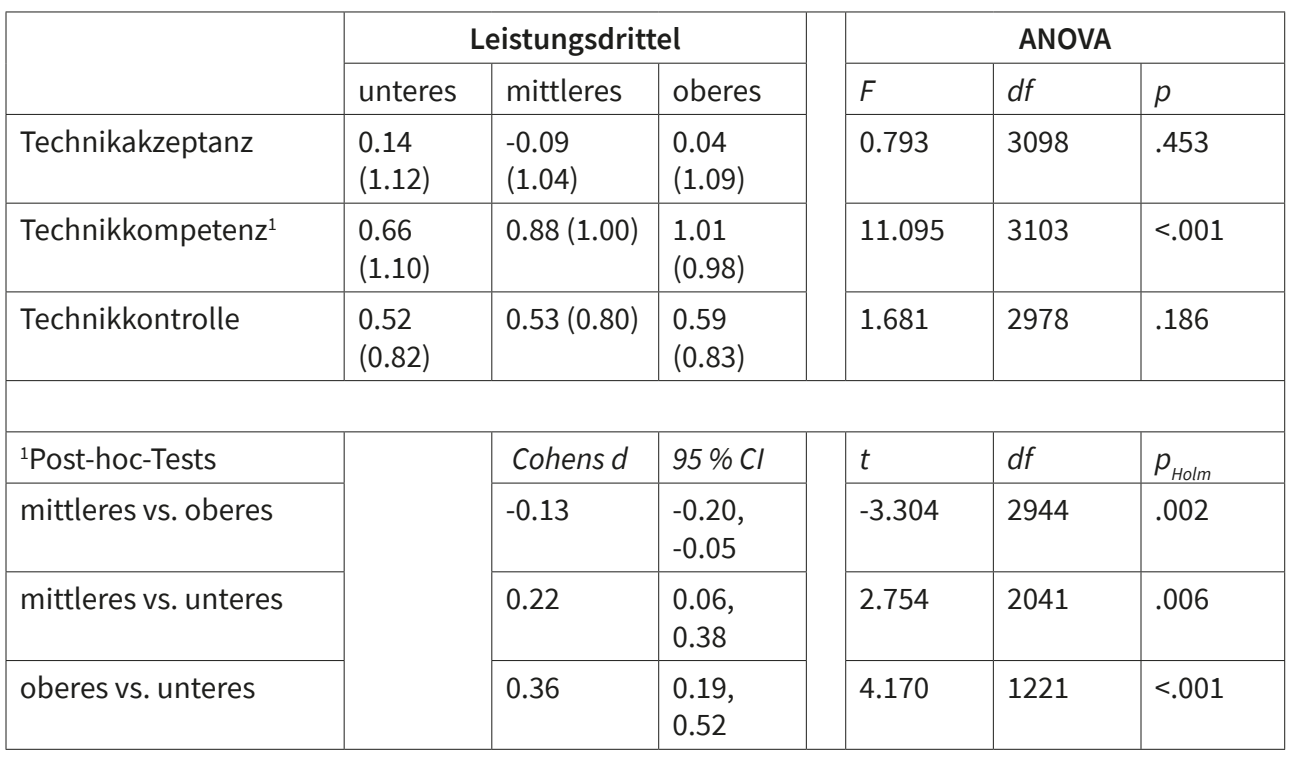

Tab. 5.: Mittelwerte der Skalen zur Technikbereitschaft nach Leistungsdritteln (Standardabweichungen in Klammern notiert, Korrektur für multiples Testen bei Post-hoc-Test nach Bonferroni-Holm-Methode).

\subsection{Vorteile und Nachteile digitaler Lernwelten}

In Anlehnung an Karapanos, Borchert und Schneider (2017) wurde zu insgesamt zehn Aspekten (5 Vorteile und 5 Nachteile) des Online-Lernens erhoben, wie stark diese im Sommersemester als vorteilhaft bzw. nachteilig erlebt wurden (Skala: $0=$ kein Vorteil/Nachteil, 4 = ein immenser Vorteil/Nachteil). Die Nachteile scheinen im Ergebnis die Vorteile zu überwiegen (siehe Tabelle 6). Vor allem fehlende soziale Interaktionen mit Peers, Dozentinnen und Dozenten sowie ein Mangel an lernbezogener Interaktion, z. B. Fragen stellen und mündliches Diskutieren, wurde als nachteilig erlebt. Aber auch der Zwang zur Selbstdisziplin und -organisation behagte den Studierenden wenig. Grösster Vorteil waren Zeit- und Kostenersparnisse durch das Lernen von zuhause sowie die grösseren Gestaltungsmöglichkeiten des eigenen Lernprozesses. 


\begin{tabular}{|l|l|c|c|c|}
\hline Rang & \multicolumn{1}{|c|}{ Item } & Kategorie & $M$ & $S D$ \\
\hline 1 & persönlich soziale Interaktion mit Peers & Nachteil & 3.22 & 1.18 \\
\hline 2 & fehlende lernbezogene Interaktion & Nachteil & 2.99 & 1.25 \\
\hline 3 & persönliche soziale Interaktion mit Dozierenden & Nachteil & 2.71 & 1.27 \\
\hline 4 & Notwendigkeit zur Selbstdisziplin und -organisation & Nachteil & 2.26 & 1.41 \\
\hline 5 & Zeit- und Kostenersparnisse & Vorteil & 2.08 & 1.56 \\
\hline 6 & eigene Gestaltung des Lernprozesses & Vorteil & 2.00 & 1.41 \\
\hline 7 & technische Hürden & Nachteil & 2.05 & 1.31 \\
\hline 8 & Bequemlichkeit und Komfort (Studieren von zuhause) & Vorteil & 1.95 & 1.48 \\
\hline 9 & Verlässlichkeit und Unabhängigkeit von Dritten & Vorteil & 1.29 & 1.36 \\
\hline 10 & didaktische Qualität bereitgestellter Materialien & Vorteil & 0.80 & 1.10 \\
\hline
\end{tabular}

Tab. 6.: Vor- und Nachteile digitaler Lernwelten (Skalenwertebereich von $0=$ kein Vorteil/Nachteil bis 4 = ein immenser Vorteil/Nachteil).

\subsection{Konzentration auf das Studium}

Den Befragten fiel es bedingt durch die Coronasituation im Sommersemester schwerer, sich auf ihr Studium zu konzentrieren $(M=-1.51, S D=2.21$; Skala: -4 (sehr viel schwerer) bis +4 (sehr viel leichter)). Während nur $10 \%$ keinen Unterschied bemerkten, berichteten etwa $71 \%$, dass es ihnen schwerer falle. Etwa jede(r) Fünfte (19\%) konnte sich hingegen besser auf das Studium konzentrieren. Die Abweichung von der Skalenmitte $(M=0)$ ist moderat $(t(3401)=-39.947, p<001, d=-0.69,95 \% \mathrm{Cl}[-0.72$, $-0.65])$. Unterschiede zwischen unterem, mittlerem und oberem Leistungsterzil sind hingegen nicht festzustellen $(F(2,3141)=0.279, p=.756)$. Ob sich Studierende leichter oder schwerer auf ihr Studium konzentrieren konnten, lässt sich besser auf Basis ihrer Einschätzungen zu Vor- und Nachteilen digitaler Lernwelten erklären (siehe 3.10). In einer linearen Regression (siehe Tabelle 7) erklären die 10 erhobenen Aspekte $50 \%$ der Varianz (adj. $\left.R^{2}, F(10,3149)=315.362, p<.001\right)$. Die Einschätzungen, inwieweit die Lernautonomie als vorteilhaft, aber auch die daraus erwachsende Notwendigkeit zur Selbstdisziplin und -organisation als nachteilig erlebt werden, stellen die beiden stärksten Prädiktoren im Modell dar. Alle zehn Prädiktoren unterschreiten auch nach Korrektur für multiples Testen nach der Bonferroni-Holm-Methode das notwendige Signifikanzniveau. Neun der zehn Regressionskoeffizienten besitzen erwartungskonforme Vorzeichen. Lediglich die eigentlich als Nachteil bewertete fehlende soziale Interaktion mit Peers weist ein positives Vorzeichen auf. Auch wenn die Vermutung plausibel erscheint, dass besonders gesellige Studierende, denen der Umgang mit ihren Peers fehlt, sich nun besser auf ihr Studium konzentrieren konnten, so handelt es sich hier sehr wahrscheinlich um ein statistisches Artefakt. Dafür spricht auch der negative Korrelationskoeffizient $(r=-.31, p<.001,95 \% \mathrm{Cl}[-.28$, $-.34]$ ) in der bivariaten Testung. 


\begin{tabular}{|c|c|c|c|c|c|c|}
\hline Kategorie & & $B$ & $S E$ & $\beta$ & $t$ & $p_{\text {Holm }}$ \\
\hline & (Intercept) & -0.473 & 0.134 & & -3.530 & $<.001$ \\
\hline Nachteil & $\begin{array}{l}\text { Notwendigkeit zur Selbst- } \\
\text { disziplin und -organisation }\end{array}$ & -0.529 & 0.023 & -0.336 & -23.452 & $<.001$ \\
\hline Vorteil & $\begin{array}{l}\text { eigene Gestaltung des Lern- } \\
\text { prozesses }\end{array}$ & 0.334 & 0.027 & 0.214 & 12.261 & $<.001$ \\
\hline Nachteil & $\begin{array}{l}\text { fehlende lernbezogene } \\
\text { Interaktionen }\end{array}$ & -0.202 & 0.030 & -0.114 & -6.813 & $<.001$ \\
\hline Vorteil & $\begin{array}{l}\text { didaktische Qualität bereit- } \\
\text { gestellter Lernmaterialien }\end{array}$ & 0.185 & 0.032 & 0.091 & 5.750 & $<.001$ \\
\hline Nachteil & technische Hürden & -0.135 & 0.024 & -0.08 & -5.725 & $<.001$ \\
\hline Nachteil & $\begin{array}{l}\text { persönliche soziale Interak- } \\
\text { tion mit Dozierenden }\end{array}$ & -0.150 & 0.031 & -0.086 & -4.846 & $<.001$ \\
\hline Vorteil & $\begin{array}{l}\text { Bequemlichkeit und Kom- } \\
\text { fort }\end{array}$ & 0.115 & 0.027 & 0.077 & 4.298 & $<.001$ \\
\hline Vorteil & Zeit- und Kostenersparnis & 0.062 & 0.023 & 0.043 & 2.630 & 0.009 \\
\hline Vorteil & $\begin{array}{l}\text { Verlässlichkeit und Unab- } \\
\text { hängigkeit von Dritten }\end{array}$ & 0.067 & 0.026 & 0.041 & 2.545 & 0.011 \\
\hline Nachteil & $\begin{array}{l}\text { persönliche soziale Interak- } \\
\text { tion mit Peers }\end{array}$ & 0.063 & 0.030 & 0.033 & 2.063 & 0.039 \\
\hline
\end{tabular}

Tab. 7.: $\quad$ Regression auf Vor- und Nachteile digitaler Lernwelten.

\section{Diskussion}

In Anbetracht der Umstände, unter denen Studium und Lehre im Sommersemester 2020 stattfanden, kann eine vorsichtig positive Bilanz gezogen werden. Die meisten Lehrveranstaltungen starteten planmässig oder mit Verspätung. Nur wenige fielen komplett aus. Den befragten Studierenden war es somit weitgehend möglich, ihr Studium wie geplant fortzuführen. Eine Mehrheit verfügte dabei über die notwendigen technischen Voraussetzungen, an digitaler Lehre teilzunehmen, wenngleich eine nicht zu vernachlässigende Minderheit punktuell Probleme berichtete. Vor allem stabile Internetverbindungen mit ausreichender Bandbreite für datenintensive Dienste wie Videotelefonie und grosse Downloads scheinen keine Selbstverständlichkeit zu sein. Nach Zahlen des Bundesministerium für Verkehr und digitale Infrastruktur verfügen in Sachsen $90.6 \%$ der Haushalte über einen Breitbandanschluss mit mindestens $16 \mathrm{Mbit} / \mathrm{s}$ Bandbreite und damit etwas weniger als im Bundesdurchschnitt mit 95.4 \% (BMVI, 2019). Die immer wieder bemängelte unzureichende Verfügbarkeit von Breitbandinternet erklärt dieses Defizit also nur teilweise. Möglicherweise beginnt das Problem auch erst hinter der Anschlussdose, wo einer immer grösseren Zahl an WLAN-fähigen Geräten keine ausreichende Netzwerktechnik gegenübersteht. Dieses Problem kann sich für Studierende noch verstärken, denn nach den Daten der 21. Sozialerhebung wohnen aktuell nur $21 \%$ der Studierenden alleine (Middendorff u. a. 2017). Wird sogar mit den Kommilitoninnen und Kommilitonen der Wohnraum geteilt, kann die gleichzeitige Nutzung des Internetanschlusses durch mehrere 
Personen eines Haushalts diese grösseren Probleme während des Lockdowns möglicherweise erklären. Gelernt wird vorrangig mit PC und Laptop. Primär auf mobile Endgeräte setzt nur etwa jede( $r$ ) Zehnte. Jedem und jeder Siebenten fehlte es an Peripheriegeräten wie Webcams und Mikrophonen. Zu berücksichtigen ist aber, dass gerade in der Anfangszeit des Lockdowns durch die Ausweitung von Heimarbeit die Nachfrage sprunghaft anstieg und es damit im Handel insbesondere bei Webcams zu Engpässen kam, Geräte also entweder gar nicht oder nur zu horrenden Preisen bezogen werden konnten (Kluczniok 2020). Da absehbar auch im Sommersemester 2021 Lehrveranstaltungen noch online stattfinden werden, sind Dozentinnen und Dozenten gut beraten, Voraussetzungen zu Beginn zu erheben und ihr Lehrkonzept entsprechend anzupassen. Im Sommersemester 2020 war das eher die Ausnahme als die Regel.

Technisch setzten Dozentinnen und Dozenten im Sommersemester stark auf Learning-Management-Systeme, denen die befragten Studierenden eine - wenn auch nicht optimale aber immerhin - akzeptable Gebrauchstauglichkeit bescheinigten. Das Lerngeschehen wurde vor allem von digitalen Texten und Präsentationen dominiert. Kollaborative oder interaktive Medientypen kamen eher selten zum Einsatz. Das legt die Vermutung nahe, dass Hochschullehre vielfach als «Contentverabreichung> praktiziert wurde. Aus didaktischer Sicht mag Hochschullehre damit hinter ihren Möglichkeiten bleiben. Die befragten Studierenden zeigten sich in der Tendenz aber damit zufrieden - möglicherweise auch deswegen, weil das Lernen mit Texten und Präsentationen eine gute Passung zu gefestigten Lerngewohnheiten aus dem Offline-Studium aufwies und eine höhere Autonomie hinsichtlich der eigenen Zeiteinteilung ermöglichte.

Lernaktivitäten und Studienorganisation bereiteten den Studierenden deutliche grössere Schwierigkeiten als in den Semestern zuvor. Auch fiel es ihnen grundsätzlich schwerer, sich auf ihr Studium zu konzentrieren. Zwar wurden z. B. Zeit- und Kosteneinsparungen des Lernens von zuhause und mehr Autonomie bei der Gestaltung des eigenen Lernprozesses als vorteilhaft erlebt, Letzteres erfordert dann aber auch Selbstdisziplin und -organisation. Insgesamt scheinen die Nachteile zu überwiegen. Wenig überraschend war es die fehlende soziale Interaktion mit Peers, die für die Befragten den grössten Nachteil darstellte. Ein Studium ist offensichtlich mehr als die Summe belegter Module. Erst an zweiter Stelle rangierte die fehlende lernbezogene Interaktion, also bspw. die Zwischenfrage in der Vorlesung oder die Diskussion im Seminar.

Die häufig geäusserte Vermutung, besonders die leistungsschwächeren Studierenden würden durch die veränderten Studienbedingungen in der Coronazeit besonders belastet, lässt sich mit den Daten dieser Untersuchung nicht untermauern. Weder bei der Bewältigung von Lernaktivitäten, bei der Studienorganisation noch bei der Fähigkeit, sich auf das Studium zu konzentrieren, gibt es bedeutsame 
Gruppenunterschiede. Lediglich mit den angebotenen Lernmedien zeigte sich das untere Leistungsdrittel etwas unzufriedener als die beiden anderen. Ob dies aber ein Effekt der veränderten Studienbedingungen ist oder dieser auch unabhängig davon auftritt, muss offenbleiben. Unberücksichtigt bleiben in einer solchen Betrachtung Randeffekte. Bereits kleine Mittelwertunterschiede können zwischen zwei oder mehr Gruppen dazu führen, dass an den Rändern der Verteilung deutliche Disparitäten zwischen diesen entstehen. So gehören in der vorliegenden Stichprobe von den Studierenden, die die grössten Schwierigkeiten mit der Organisation ihres Studiums im Vergleich zum Vorsemester berichten ${ }^{4}$, fast $11 \%$ zum unteren Leistungsdrittel, obwohl diese nur $5 \%$ der Gesamtstichprobe ausmachen. Der geringe Anteil an Studierenden des unteren Leistungsdrittels unter den Befragten und die Kategorisierung über eine Selbsteinschätzung stellen beachtliche Einschränkungen dar, weshalb die hierzu getroffenen Aussagen mit Vorbehalt aufzunehmen sind.

Technikbereitschaft erwies sich in seiner Bedeutung als nachrangig. Keine der drei Facetten zeigte nennenswerte Zusammenhänge mit anderen erfassten Merkmalen. Generell - so ist zu vermuten - scheinen Studierende ausreichend technikbereit zu sein, sodass die verbleibende Varianz kaum noch Unterschiede bei der Bewältigung des Studiums erklärt. Dies deckt sich mit der Tatsache, dass - gemessen an anderen Nachteilen digitaler Lernwelten - technische Hürden als am wenigsten relevant bewertet wurden. Vielmehr sind es spezifische Kompetenzen wie etwa die Erstellung eigener Medien, die Verfügbarkeit digitaler Fachliteratur oder Defizite in der technischen Ausstattung, die einigen das digitale Studium noch erschweren. Allerdings gilt es zu berücksichtigen, dass der methodische Zugang - Onlinebefragung - als Filter wirkte und so vorwiegend technisch versierte Studierende in das Sample gelangten.

\section{Resümee und Ausblick}

Mit aller Vorsicht, die bei der Interpretation von Umfragedaten geboten ist, können die sächsischen Hochschulen in puncto digitaler Lehre alles in allem zufrieden sein mit dem, was im Sommersemester 2020 in sehr kurzer Zeit geleistet wurde. Der Lehrbetrieb wurde weitgehend aufrechterhalten, sodass Studierende ihr Studium im Allgemeinen fortführen konnten. Schwierigkeiten bei der Organisation des eigenen Studiums fielen moderat aus. Lernaktivitäten waren deutlicher von den veränderten Studienbedingungen betroffen. Überwiegend zufrieden zeigten sich die befragten Studierenden mit den angebotenen Lernmedien, wenngleich eine gewisse Monotonie aus digitalen Präsentationen und Texten erkennbar wird. Dies ist aber kein Vorwurf an die Kolleginnen und Kollegen, ging es doch im ersten digitalen Semester oft unter zusätzlicher Belastung durch die Zuhause-Beschulung der eigenen Kinder

4 Individueller Messwert = Skalenminimum 
bei vielen ums «reine Überleben〉. Potential bietet vor allem das Medium Video, mit dem Studierende offenbar gern lernen, das aber noch selten zum Einsatz kommt. Videos müssen nicht immer selbst produziert werden - ein in der Tat aufwendiges Unterfangen. Onlinevideoplattformen bieten eine grosse Zahl teils ausgezeichneter Instruktionsvideos zu einer Vielzahl von Themen. Gerade Dozentinnen und Dozenten in Einführungsveranstaltungen ‘klassischer Fächer könnten schnell fündig werden.

Die Vor- und Nachteile digitaler Lernwelten traten selten offensichtlicher zum Vorschein als im Sommersemester 2020. Ja, Studierende schätzen das bequeme Studieren von zuhause, die Zeitersparnis, weil die Fahrt zum Campus entfällt und die grösseren Freiheiten bei der Gestaltung des eigenen Lernprozesses. Gleichzeitig vermissen sie aber auch den Kontakt zu Kommilitoninnen und Kommilitonen, den direkten Austausch mit ihren Dozentinnen und Dozenten und die soziale Verbindlichkeit von Präsenzlehre. Damit bestätigt sich die in der Befragung von Pauli et al. (2020) deutlich gewordene Befürchtung eines unpersönlicheren Studiums. Auch hierzu braucht es Lösungen, diese offenbar wichtigen Facetten eines Studiums auch in digitalen Lehrkonzepten stärker zu berücksichtigen und Lernanlässe zu schaffen, die auch aus der Distanz Gemeinschaft und soziales Miteinander stiften.

In ihrer Strategie zur Digitalisierung in der Hochschulbildung (SMWK 2018) formulierten Staatsregierung und Hochschulen in Sachsen vier Ziele für den Einsatz digitaler Werkzeuge in der Hochschullehre: 1) eine Verbesserung der Lehrqualität, 2) eine Steigerung des Studienerfolges, 3) die Stärkung der Wettbewerbsfähigkeit der Hochschulen und 4) eine bessere internationale Sichtbarkeit. "Digitale Lehrangebote», war man sich sicher, «werden auch zukünftig nicht vollständig an die Stelle der Präsenzlehre treten» (SMWK 2018, 3). Dass diese hier angesprochene vollständige Substitution selbst unter den erschwerten Bedingungen einer Pandemie trotzdem gelang, ist zweifelsohne ein Erfolg. Obwohl nicht explizit für diesen Fall vorgesehen, war mit den aufgebauten (LMS, E-Learning-Support-Teams an den Hochschulen, E-Book-Lizenzen usw.) und kurzfristig beschaffbaren Ressourcen (vor allem Videokonferenzdienste und -technik) eine Umstellung auf Pandemiebetrieb möglich. Gleichwohl ist damit gezeigt, dass mit den verfügbaren Mitteln auch schon vor der Coronapandemie deutlich mehr digitale Lehre möglich gewesen wäre. Bereits jetzt stellt sich die Frage, wie nach der Pandemie eine Rückkehr zum Status quo ante vermieden werden kann (z. B. Bils u. a. 2020). Die Nutzenbilanz der verfügbaren Technologien wird - und das gilt über den sächsischen Hochschulraum hinaus - dabei selten hinterfragt. Geeignete Rahmenbedingungen (Rechtsgrundlagen, Finanzierung, Infrastruktur usw.) sind immer nur als notwendig aber keinesfalls hinreichend zu verstehen. Digitale Lehre bedeutet nicht automatisch bessere Lehre, auch wenn sie häufig mit Attributen wie ‘zeitgemäss> oder ‘modern` versehen wird. Sie ersetzt (und ergänzt) nicht selten alte Probleme durch neue. Das natürliche Experiment (digitales Sommersemester> hat das sehr klar vor Augen geführt. Was in Krisenzeiten als technische 
Notlösung akzeptiert wird, kann nach der Krise bereits als inakzeptabel wieder abgewiesen werden. Insofern gilt es in Zukunft stärker als bisher, den Fokus auf die Qualität digitaler Bildungstechnologien zu richten. Seit Jahrzehnten einschlägige Technologieakzeptanzmodelle zeigen unmissverständlich, dass Technik im Privaten wie im Arbeitskontext dann nachhaltig angenommen wird, wenn sie einen wahrnehmbaren Nutzen bietet und sich dieser Nutzen wahrnehmbar einfach realisieren lässt (Davis 1989; Yousafzai, Foxall, und Pallister 2007). Das gilt auch für Bildungstechnologien (Šumak, Hericko, und Pušnik 2011). Erst wenn Bildungstechnologien einen positiven Netto-Nutzen aufweisen und sich auch gegenüber alternativen Handlungsoptionen als vorteilhaft erweisen, ist von einer dauerhaften freiwilligen Annahme durch Lehrende und Lernende auszugehen. Die gemessen an kommerziellen Onlinewerkzeugen unterentwickelte Usability bei LMS weist auch Defizite in diesem Punkt hin. Modelle der Mensch-Technik-Interaktion benennen zudem die Befriedigung psychogener Bedürfnisse als wichtiges Akzeptanzkriterium (Diefenbach, Kolb, und Hassenzahl 2014; Kolb, und Hassenzahl 2014). Soziale Eingebundenheit, die Studierenden im Sommersemester so sehr fehlte, ist bspw. eines davon. In vielen Lebensbereichen haben es digitale Technologien geschafft, ihre analogen Konkurrenten abzulösen. In der Bildung gelang das erst unter den Bedingungen einer Pandemie. Vielleicht wäre es erfolgversprechender, sich zukünftig der Frage zuzuwenden, warum die Entwicklung in der Bildung so gänzlich anders verlief. Die daraus resultierenden Erkenntnisse könnten hilfreicher sein, als jedes weitere Strategiepapier.

\section{Literatur}

Bangor, Aaron, Philip Kortum, und James Miller. 2009. «Determining what individual SUS scores mean: Adding an adjective rating scale». Journal of Usability Studies 4 (3): 114-123. https:// uxpajournal.org/determining-what-individual-sus-scores-mean-adding-an-adjective-ratingscale/.

Bils, Annabell, Barbara Braun, Toni Bünemann, Tina Scheuring, Carolin Sutter, Verena Meyer, Sandra Neuner, Barbara Wagner, und Yasemin Wistuba. 2020. «Corona-Semester 2020 - Ad-hocMaßnahmen evaluieren und nachhaltig verankern». Diskussionspapier 11, Berlin: Hochschulforum Digitalisierung.

BMVI. 2019. «Bericht zum Breitbandatlas Teil 1: Ergebnisse (Stand Ende 2019)». Berlin.

Bond, Melissa, Victoria I. Marín, Carina Dolch, Svenja Bedenlier, und Olaf Zawacki-Richter. 2018. «Digital Transformation in German Higher Education: Student and Teacher Perceptions and Usage of Digital Media». International Journal of Educational Technology in Higher Education 15 (1): 48. https://doi.org/10.1186/s41239-018-0130-1.

Brooke, John. 1996. «SUS - a 〈quick and Dirty> Usability Scale». In Usability Evaluation in Industry, herausgegeben von Patrick W. Jordan, B. Thomas, Ian Lyall McClelland, und Bernard Weerdmeester, 189-94. London: Taylor \& Francis. 
Crawford, Joseph, Kerryn Butler-Henderson, Jürgen Rudolph, Matt Glowatz, Rob Burton, Paola Magni, und Sophia Lam. 2020. «COVID-19: 20 Countries« Higher Education Intra-Period Digital Pedagogy Responses». Journal of Applied Teaching and Learning (JALT) 3 (1). https://doi. $\operatorname{org} / 10.37074 / j a l t .2020 .3 .1 .7$.

Davis, Fred D. 1989. «Perceived usefulness, perceived ease of use, and user acceptance of information technology». MIS Quarterly 13 (3): 319-40. https://doi.org/10/cc6.

Diefenbach, Sarah, Nina Kolb, und Marc Hassenzahl. 2014. «The 〈hedonic in human-computer interaction: history, contributions, and future research directions». In Proceedings of the 2014 Conference on Designing Interactive Systems, 305-314. DIS 14. New York, NY: ACM. https://doi. org/10/gf4zv4.

Döring, Nicola, und Jürgen Bortz. 2016. Forschungsmethoden und Evaluation in den Sozial- und Humanwissenschaften. 5. Aufl. Springer-Lehrbuch. Berlin: Springer.

Feierabend, Sabine, Thomas Rathgeb, und Theresa Reuter. 2019. "JIM-Studie 2019. Jugend, Information, Medien. Basisuntersuchung zum Medienumgang 12- bis 19-Jähriger.» Stuttgart: Medienpädagogischer Forschungsverbund Südwest.

Grosch, Michael, und Gerd Gidion. 2011. Mediennutzungsgewohnheiten im Wandel: Ergebnisse einer Befragung zur studiumsbezogenen Mediennutzung. Karlsruhe: KIT Scientific Publishing.

$\mathrm{Hu}$, Li-tze, und Peter M. Bentler. 1999. «Cutoff criteria for fit indexes in covariance structure analysis: Conventional criteria versus new alternatives». Structural Equation Modeling: A Multidisciplinary Journal 6 (1): 1-55. https://doi.org/10/dbt.

Huber, Stephan Gerhard, und Christoph Helm. 2020. «COVID-19 and Schooling: Evaluation, Assessment and Accountability in Times of Crises-Reacting Quickly to Explore Key Issues for Policy, Practice and Research with the School Barometer». Educational Assessment, Evaluation and Accountability 32 (2): 237-70. https://doi.org/10.1007/s11092-020-09322-y.

Jänsch, V. K., und E. Bosse. 2018. Messinstrument für die Wahrnehmung von Studienanforderungen (MWS). Zusammenstellung sozialwissenschaftlicher Items und Skalen (ZIS).

Karapanos, Marios, Thomas Borchert, und André Schneider. 2017. «The adoption of educational technology from the perspective of behavioral reasoning theory». In EDULEARN17 Proceedings, herausgegeben von L. Gómez Chova, A. López Martínez, und I. Candel Torres, 88-95. Valencia: IATED. https://doi.org/10.21125/edulearn.2017.1020.

Karapanos, Marios, und Jan Fendler. 2015. «Lernbezogenes Mediennutzungsverhalten von Studierenden der Ingenieurswissenschaften. Eine geschlechterkomparative Studie.» Journal of Technical Education (JOTED) 3 (1): 39-55. https://www.journal-of-technical-education.de/index.php/joted/article/view/33.

Kluczniok, Jan. 2020. «Coronavirus: Webcams sind das neue Klopapier». netzwelt. 9. April 2020. https://www.netzwelt.de/news/177690-coronavirus-webcams-neue-klopapier.html.

Kortum, Philip, und Aaron Bangor. 2013. «Usability ratings for everyday products measured with the system usability scale». International Journal of Human-Computer Interaction 29 (2): 6776. https://doi.org/10.1080/10447318.2012.681221.

Kuncel, Nathan R., Marcus Credé, und Lisa L. Thomas. 2005. «The Validity of Self-Reported Grade Point Averages, Class Ranks, and Test Scores: A Meta-Analysis and Review of the Literature». Review of Educational Research 75 (1): 63-82. https://doi.org/10.3102/00346543075001063. 
Middendorff, Elke, Beate Apolinarski, Karsten Becker, Philipp Bornkessel, Tasso Brandt, Sonja Heißenberg, und Jonas Poskowsky. 2017. «Die wirtschaftliche und soziale Lage der Studierenden in Deutschland 2016: 21. Sozialerhebung des Deutschen Studentenwerks durchgeführt vom Deutschen Zentrum für Hochschul- und Wissenschaftsforschung». Bonn, Berlin: Bundesministerium für Bildung und Forschung.

Neyer, Franz J., Juliane Felber, und Claudia Gebhardt. 2012. «Entwicklung und Validierung einer Kurzskala zur Erfassung von Technikbereitschaft». Diagnostica 58 (2): 87-99. https://doi. org/10.1026/0012-1924/a000067.

Pauli, Paul, Silke Neuderth, und Maria Schuppert. 2020. Studieren in Corona-Zeiten. Pressemitteilung der Universität Würzburg. https://www.uni-wuerzburg.de/aktuelles/pressemitteilungen/ single/news/studieren-in-coronazeiten-1/.

Persike, Malte, und Julius-David Friedrich. 2016. Lernen mit digitalen Medien aus der Studierendenperspektive. Sonderauswertung aus dem CHE Hochschulranking für die deutschen Hochschulen. Essen: Edition Stifterverband.

Rauer, Matthias. 2011. «Quantitative Usability-Analysen mit der System Usability Scale (SUS)». Seibert Media Weblog (blog). 2011. https://blog.seibert-media.net/blog/2011/04/11/usablility-analysen-system-usability-scale-sus/.

Richter, Constanze, und Philip Dunkhase. 2020. Online-Umfrage: 91\% der Studierenden haben Sorgen zum ersten Corona-Semester. Pressemitteilung der Hochschule Aalen. https://www. hs-aalen.de/de/news/3335.

Sibley, Chris G., Lara M. Greaves, Nicole Satherley, Marc S. Wilson, Nickola C. Overall, Carol H. J. Lee, Petar Milojev, u. a. 2020. "Effects of the COVID-19 pandemic and nationwide lockdown on trust, attitudes toward government, and well-being.» American Psychologist 75 (5): 618. https://doi.org/10.1037/amp0000662.

SMWK. 2018. «Strategie zur Digitalisierung in der Hochschulbildung». Dresden.

Statistisches Landesamt Sachsen. 2019. «Studierende an den Hochschulen im Freistaat Sachsen. 2018». Statistischer Bericht. B III 1-j/18.

Šumak, Boštjan, Marjan Hericko, und Maja Pušnik. 2011. «Factors affecting the adoption of eLearning: A meta-analysis of existing knowledge». In The Third International Conference on Mobile, Hybrid, and Online Learning, herausgegeben von Karlsson Göran, Dumitru Dan Burdescu, und Bernd Krämer, 31-35. Red Hook, NY: Curran Associates.

Wößmann, Ludger, Vera Freundl, Elisabeth Grewenig, Philipp Lergetporer, Katharina Werner, und Larissa Zierow. 2020. «Bildung in der Coronakrise: Wie haben die Schulkinder die Zeit der Schulschließungen verbracht, und welche Bildungsmaßnahmen befürworten die Deutschen?» ifo Schnelldienst. ifo Institut.

Yousafzai, Shumaila Y., Gordon R. Foxall, und John G. Pallister. 2007. «Technology acceptance: a meta-analysis of the TAM: Part 2». Journal of Modelling in Management 2 (3): 281-304. https:// doi.org/10.1108/17465660710834462.

Zacher, Hannes, und Cort W. Rudolph. 2020. «Individual differences and changes in subjective wellbeing during the early stages of the COVID-19 pandemic.» American Psychologist, 1-13. https://doi.org/10.1037/amp0000702. 